\title{
Mathematische Auswertung von Daten für das Wachstum von Heringen der Nordsee
}

\author{
F. KRÜGER \\ Biologische Anstalt Helgoland (Zentrale); \\ Hamburg 50, Bundesrepublik Deutschland
}

\begin{abstract}
Mathematical evaluation of growth data of the North Sea herring. The parameters of the reciprocal function ("Reziprokfunktion") for the growth of herrings (Clupea barengus) are calculated from older and more recent measurements. The logarithmic expression of the proposed reciprocal function is as follows: $\log y_{x}=\log y_{\max }-\frac{1}{\chi+\xi} \log N$. Values less than 1 are found for the additive age $(\xi)$. In further calculations 0.4 is used as the estimated mean value. Measurements made before the second world war yield ca. $30 \mathrm{~cm}$ for the maximum value $\left(\mathrm{L}_{\max }\right)$. After this period the maximum values increase to $\mathrm{ca} .34 \mathrm{~cm}$. The Scandinavian and Atlantic herrings differ from North Sea herrings by higher maximum values. The values for the constant of velocity $(\log N)$ may be different for identical $\xi$ and $Y_{\max }$ values. The velocity constant determines the position of the inflection point of the growth curve. The dimension, which is only dependent on the maximum value, is at the inflection point: $\frac{Y_{\max }}{7,389}$ From the results of Schumacher (1967) on the growth of 3 herring populations from the North Sea it was calculated that the values for the constant of velocity rise from northern to southern areas. A low value for the constant of velocity marks an early inflection point and a high velocity of growth before this point and vice versa. The growth of the 3 populations tends to almost the same maximum value; consequently, a high velocity before the inflection point is compensated by a lower velocity after this point and vice versa. The maximum velocity of linear growth at the point of inflection is given by the expression $\frac{Y_{\max }}{4,25 \cdot \log N}$. This expression may possibly be a useful device for quantitative comparisons of growth processes.
\end{abstract}

\section{EINLEITUNG}

Es ist seit langem bekannt, daß man in der Nordsee Heringsbestände findet, die sich in der Länge der einzelnen Altersstufen unterscheiden, ohne daß sich parallel hierzu morphologisch sicher faßbare Merkmale erkennen lassen.

HEINCKE (1898) vermutete das Vorliegen von Rassen, die sich durch erbliche Eigentümlichkeiten des Wachstumsablaufs unterscheiden und versuchte sie auf statistischem Wege exakt gegeneinander abzugrenzen. Seit dieser Zeit ist die Diskussion um die Frage nach der Existenz eindeutig unterscheidbarer Heringsrassen nicht abgerissen.

$\mathrm{Da}$ die statistische Auswertung nicht ganz einfach durchzuführen ist, hat man 
nach morphologischen Merkmalen gesucht - wie der Zahl der Wirbel, der Zahl der Kielschuppen, der Zahl der Kiemenreusenfortsätze usw. - um eine eindeutige Zuordnung zu bestimmten Rassen zu ermöglichen. Die Untersuchung yon Schumacher (1967) an drei Populationen des Nordseeherings hat aber gezeigt, daß die Variabilität dieser Merkmale so groß ist, daß nur bei den Kiemenreusenfortsätzen ein schwach gesicherter Unterschied zwischen der nördlichen und der südlichen Population gegeben ist.

Da sich die verschiedenen Heringspopulationen offensichtlich in ihren Wachstumseigenschaften unterscheiden, versuchte ScHumacher durch Bestimmung der Parameter der Wachstumsfunktion von v. BertalANFFY zu einer Charakterisierung zu kommen, ohne hierbei allerdings ein eindeutig auswertbares Ergebnis zu erzielen. Vor ihm hatte schon Burd (1962) die gleiche Funktion zur Beschreibung des Wachstums von von ihm gegebene Formulierung nicht den $\tau_{0}$-Wert (bzw. $L_{0}$ ) einschließt, sind seine Auswertungen nicht befriedigend.

Es ist sicher, daß die BerTalanffy-Funktion sehr gut geeignet ist, die Daten des Heringswachstums als Funktion des Alters wiederzugeben. Aber die Bestimmung der Parameter erfolgte nicht einheitlich bei den verschiedenen Autoren, und außerdem bestehen Bedenken gegen den Aussagewert ihrer Parameter (KRüger 1973). So ist es nicht verwunderlich, daß die mathematischen Auswertungen auf der Basis der BERTALANFFY-Funktion keine bemerkenswerten Aufschlüsse lieferten.

Als ersten Versuch einer mathematischen Beschreibung des Wachstums unterschiedlicher Heringspopulationen muß man die grundlegende Arbeit von Ford (1933) ansehen, in der er die an seinen Namen geknüpfte Wachstumsdarstellung veröffentlichte. Die von ihm angewandte graphische Darstellung läßt aber bei den Heringen keine sicher erkennbaren Unterschiede erwarten.

Wir wissen heute, daß die von Ford und später von WALFORD (1946) aufgefundene Wachstumsdarstellung nichts anderes ist, als eine äußerst einfach durchzuführende lineare Wiedergabe der Bertalanffy-Funktion. Zwischen den Parametern bestehen exakt definierte Beziehungen (BAgENAL 1955, HOHENDORF 1966, KRÜGER 1967).

Nun stellt die BerTalanffy-Funktion nicht die einzige Möglichkeit zur mathematischen Simulation von Wachstumsdaten dar, und aus verschiedenen Gründen kann man sie auch nicht als ein optimales Wachstumsmodell ansehen. Ein wesentlicher Nachteil der BertalAnFFY-Funktion liegt darin, daß sie keinen Wendepunkt einschließt, dessen mathematische Darstellung für das Verständnis des Heringswachstums von groBer Bedeutung ist, wie wir sehen werden.

Vor einigen Jahren hatte ich eine andere Formel vorgeschlagen (KRüGER 1962, 1965, 1967), die mindestens ebenso gute Näherungen an gegebene Wachstumsdaten gestattet und darüber hinaus eine Reihe weiterer Vorteile bietet. Abweichend von der BERTALANFFY-Funktion stellt sie die mit zunehmendem Alter abnehmenden Zuwachsraten durch einen reziproken Alterswert dar. Aus diesem Grunde bezeichne ich sie in den folgenden Ausführungen als „Reziprokfunktion“. Es erschien mir von Interesse zu prüfen, ob mit ihrer Hilfe weitergehende Aufschlüsse über die Variabilität des Heringswachstums zu gewinnen sind. 


\section{METHODIK}

Die Reziprokfunktion hat in der von mir angewandten Schreibung die Form:

$$
y_{\chi}=-\frac{Y_{\max }}{N \frac{1}{x+\xi}}
$$

In ihr ist $\mathrm{Y}_{\max }$ der Grenzwert, dem sich die Wachstumskurve asymptotisch nähert; $\mathrm{N}$ bezeichne ich als Geschwindigkeitskonstante; $\mathrm{y}_{\chi}$ ist die Dimension, die der Organismus im Alter $\chi$ erreicht und $\xi$ ist ein Zeitwert, der zum Geburtsalter $\chi$ addiert wird, um eine optimale Annäherung an die Meßdaten zu erreichen. Der Parameter $\xi$ beschreibt die Krümmung der Wachstumskurve und kann daher auch als Krümmungsparameter bezeichnet werden.

Die Grundlage der Auswertungen bildet die logarithmierte Funktion:

$$
\log y_{\chi}=\log Y_{\max }-\frac{1}{\chi+\xi} \log N
$$

Sie stellt die Gleichung einer Geraden dar und bietet auf dem Weg der linearen Regressionsberechnung eine Möglichkeit zur Berechnung der Parameter $Y_{\max }$ und $\log N$. Der optimale Wert von $\xi$ muß durch Variation von $\xi$ durch iterative Berechnung ermittelt werden. Als Kriterium für die Annäherung an die Meßdaten diente die Standardabweichung.

Bezeichnet man mit y die gemessenen und mit $\hat{y}$ die errechneten Werte, so wird bei der Regressionsauswertung der Gleichung (1b)

$$
\Sigma(\log y-\log \hat{y})^{2}=\min .
$$

Durch die Logarithmierung wird also nicht die Summe der quadrierten linearen Abweichungen zum Minimum sondern die Summe der quadrierten relativen Differenzen. Da auch der Streubereich der Meßwerte um den Mittelwert eine Funktion der jeweiligen Größe ist und der relative Streubereich eine recht konstante Größe darstellt, erscheint die relative Abweichung bei Wachstumsdarstellungen günstiger als die lineare Abweichung. Als optimal sehe ich also die Lösungen an, bei denen die Summe der quadrierten relativen Abweichungen zum Minimum wird.

Die Standardabweichung der Logarithmen, die man bei der Regressionsauswertung von Gleichung (1b) erhält, bezeichne ich mit $s_{l o g}$. Sie stellt einen wenig anschaulichen und durch eine oder zwei Nullen nach dem Komma mit höherer Stellenzahl anzugebenden Wert dar. Nun bildet die Grundlage von $s_{\text {log }}$ die Differenz:

$$
(\log y-\log \hat{y})=\log -\hat{y}^{-}
$$

Der Numerus dieses Quotienten ist also der Faktor, mit dem man $\hat{y}$ multiplizieren muß, um zu y zu kommen. Bezogen auf $\hat{y}=100$ muß man diesen Numerus mit 100 multiplizieren. Zieht man von diesem Wert $100 \mathrm{ab}$, so erhält man - wie leicht verständlich ist - eine prozentuale Abweichung, die ich mit s\% bezeichne.

$$
s \%=\left(100 . \text { num } s_{\text {log }}\right)-100
$$

Durch die Quadrierung der Differenz bei der Ermittlung der Standardabweichung 
wird es gleichwertig, ob man den Meßwert oder den theoretischen Wert als Bezugsgröße wählt.

Der auf diesem Weg berechnete Prozentwert entspricht nicht ganz den in üblicher Weise berechneten Prozentangaben. Bei den durchweg sehr niedrigen prozentualen Abweichungen fällt dieser Unterschied aber nicht ins Gewicht.

Die prozentuale Standardabweichung $\left(\mathrm{s}^{\%} \%\right)$ bietet in einfacher Weise eine Vergleichsmöglichkeit mit dem in Prozent ausgedrüdkten Variationskoeffizienten der Meßwerte. Naturgemäß darf er durch die rechnerischen Werte nicht überschritten werden.

In Tabelle 19 sind bei Trambs-Lyche (1965) die Varianzen der Meßwerte angegeben, aus denen sich die Variationskoeffizienten berechnen lassen. Im Durchschnitt ergeben sich für das Längenwachstum des Herings Variationskoeffizienten von etwa $4 \%$. Hiermit ist die Grenze für die prozentuale Standardabweichung gegeben, die sich noch mit den Meßwerten vereinbaren läßt. Sie wurde in keinem Falle überschritten. Ober den Variationskoeffizienten der Gewichtsangaben fand ich keinen Hinweis. Er liegt sicher wesentlich höher.

Soweit ich Werte für die Parameter der BerTalanffy-Funktion angebe, beruhen sie auf der Berechnung über die FORD-WALFORD-Formel (KRÜGER 1973).

\section{DER ADDITIVE ALTERSWERT FÚR DAS HERINGSWACHSTUM}

Der additive Alterswert $\xi$ bildet in der Reziprokfunktion den entscheidenden Parameter für die Darstellung des Verlaufs der Wachstumskurven. Bei den Heringen stößt seine eindeutige Bestimmung auf gewisse Schwierigkeiten. Es liegen zwar in der Literatur zahlreiche Daten über das Heringswachstum vor, sie beginnen aber in den meisten Fällen erst mit den Altersstufen 2 oder 3. Da aber beim Hering die stärkste Größenzunahme vor diesem Zeitpunkt liegt, umfassen diese Angaben nur eine relativ geringe Wachstumsspanne. Zwischen den Altersstufen 0 und 3 liegt der Bereich der stärksten Krümmung der Wachstumskurve, der für eine genaue Bestimmung des $\xi$ Wertes besonders wichtig ist. Nach dieser Zeit sind die Kurven nur schwach gekrümmt und machen dadurch die Bestimmung des Krümmungsparameters $\xi$ unsicher.

Aus diesem Grunde erscheint die bekannte Arbeit von Ford (1933) als Ausgangspunkt geeignet, da sie eine Reihe von Tabellen enthält, die mit der Altersstufe 0 beginnen. Sie umfassen das Längenwachstum von Heringen sehr verschiedener Herkunft. Die aus ihren Zahlen sich ergebenden optimalen Parameterwerte sind in Tabelle $1 \mathrm{zu-}$ sammengestellt.

Die Wachstumskurven dieser Heringsbestände sind übereinstimmend dadurch ge-kennzeichnet, daß sich für sie $\xi$-Werte ergeben, die unter 1 liegen. Hierin kommt die schon erwähnte hohe Wachstumsgeschwindigkeit in den ersten Lebensjahren zum Ausdruck. Eine vollkommene Ubereinstimmung der errechneten $\xi$-Werte war angesichts der unvermeidlichen Unschärfe der Wachstumsdaten kaum zu erwarten. So muß man sich mit der Größenordnung des Krümmungsparameters zufriedengeben.

Die beiden anderen Parameter der Funktion: $Y_{\max }$ und $\log N$ sind abhängig vom eingesetzten $\xi$-Wert, können also nur unter der Bedingung verglichen werden, daß ihnen der gleiche $\xi$-Wert zugrunde liegt. Diese Voraussetzung ist bei den in Ta- 
Tabelle 1

Optimale Parameter für das Wachstum von Heringen nach Tabellen aus der Arbeit von Ford (1933)

\begin{tabular}{|lrrcc|}
\hline Bezeichnung & $\xi$-Werte & $\mathrm{L}_{\max }$ & $\log \mathrm{N}$ & \multicolumn{1}{c}{$s \%$} \\
\hline Gilson & $-0,02$ & 30,04 & 0,4701 & 0,91 \\
Type Cotier & 0,15 & 30,46 & 0,4699 & 0,73 \\
Type Atlantique & 0,11 & 30,97 & 0,4377 & 0,26 \\
HJORT a & 0,05 & 33,51 & 0,4461 & 0,48 \\
HjORT b & $-0,86$ & 31,95 & 0,2599 & 1,35 \\
HJORT Island & 0,6 & 45,38 & 1,1565 & 2,01 \\
\hline
\end{tabular}

belle 1 gegebenen Werten nicht gegeben. Immerhin weisen die $\mathrm{L}_{\max }$-Werte für die drei ersten Beispiele eine erstaunliche Übereinstimmung auf. Demgegenüber weichen die anderen Maximalwerte stärker ab.

Nun stammen die Messungen der Tabelle 1 aus der Zeit vor dem 2. Weltkrieg. Es ist aber bekannt (PARrish \& SAville 1967), daß die Heringsbestände der Nordsee in den letzten Jahrzehnten eine erhebliche Größenzunahme erfahren haben. Es war daher erforderlich, aus neuerer Zeit stammende Meßreihen auszuwerten, um zu erkennen, in welcher Weise sich diese Größenzunahme auf die Wachstumsparameter ausgewirkt hat.

Aus den Statistical News Letters Nr. 31 von 1965 (Thambs-Lyche) habe ich solche Tabellen ausgewählt, die die Altersgruppe 0 oder wenigstens die Altersgruppe 1 einschließen. Das Ergebnis der Auswertungen ist in Tabelle 2 zusammengestellt.

Auch aus diesen Meßreihen ergeben sich fast durchweg $\xi$-Werte, die unter 1 liegen. Bei den Heringen von Egersund liefert zwar die ganze Zahlenreihe einen $\xi$-Wert von 1,5 , läßt man aber die 0 -Gruppe unberücksichtigt, so erhält man den "normalen“

Tabelle 2

Zusammenstellung der aus den Tabellen von Thambs-Lyche (1965) erhaltenen Parameter für das Längenwachstum von Heringen

\begin{tabular}{|lcccccc|}
\hline \multicolumn{1}{c}{ Herkunft } & Tabelle & Seite & $\xi$-Werte & $\mathrm{L}_{\max }$ & $\log \mathrm{N}$ & \multicolumn{1}{c}{${ }^{2} \% / \mathrm{s}$} \\
\hline Schottland & 48 & 58 & 0,63 & 32,91 & 0,2668 & 2,48 \\
Schottland & 49 & 59 & 0,14 & 33,70 & 0,2566 & 1,36 \\
North Minch & 51 & 62 & 1,48 & 33,50 & 0,3588 & 1,77 \\
South Minch & 52 & 66 & 0,83 & 32,74 & 0,2788 & 2,25 \\
Hebriden & 33 & 33 & 0,80 & 32,54 & 0,2493 & 1,63 \\
Island & 35 & 34 & $-0,34$ & 35,00 & 0,2901 & 2,15 \\
Norwegen & 41 & 39 & 0,57 & 33,98 & 0,2460 & 3,83 \\
Norwegen & 38 & 37 & 0,61 & 35,88 & 0,3779 & 4,36 \\
Egersund (ohne $\left.1_{0}\right)$ & 26 & 27 & 0,12 & 34,11 & 0,2156 & 2,84 \\
Skagerrak & 19 & 18 & 0,30 & 36,45 & 0,4721 & 1,99 \\
Skagerrak & 27 & 30 & 0,66 & 33,34 & 0,2537 & 1,39 \\
Bressay Shoal & 21 & 22 & $-0,01$ & 33,30 & 0,1990 & 1,31 \\
Doggerbank & 24 & 24 & 0,18 & 32,25 & 0,2259 & 3,14 \\
Fladengrund & 22 & 22 & $-0,1$ & 32,36 & 0,1915 & 2,79 \\
\hline
\end{tabular}


Wert von 0,45. Durch die Maschenselektion besteht bei den jüngsten Stadien immer die Gefahr, daß die ermittelten Durchschnittswerte zu hoch ausfallen, wodurch sich bei der Berechnung zu hohe $\xi$-Werte ergeben.

Vielleicht liefern die neueren Messungen im Schnitt etwas höhere $\xi$-Werte gegenüber Tabelle 1, man kann aber nicht von einer eindeutigen Änderung sprechen. Es bleibt immerhin erstaunlich, daß die Meßreihen so unterschiedlicher Herkunft rechnerisch eine so weitgehende Ubbereinstimmung zeigen. Sie weist auf einen prinzipiell ähnlichen Verlauf der Wachstumskurven hin, der eben in dem Krümmungsparameter $\xi$ seinen Ausdruck findet. Eine erhebliche Anderung des $\xi$-Wertes ist also mit der GröBenzunahme der Heringe nicht eingetreten. Demgegenüber liegen die Maximalwerte bei den neueren Fängen aus der Nordsee durchweg höher als bei den älteren Messungen. Die Werte für die Geschwindigkeitskonstanten - $\log \mathrm{N}$ - erfordern weiter unten eine eingehendere Behandlung.

Der additive Alterswert $\xi$ zeichnet sich durch die günstige Eigenschaft aus, daß er nicht sehr kritisch ist, so daß man auch bei Werten, die vom mathematischen Optimum abweichen, noch einen befriedigenden Anschluß an gegebene Meßdaten erreicht. Eine Kontrollmöglichkeit ist in der Ermittlung der Standardabweichung gegeben.

Angesichts der unvermeidlichen Unsicherheit der Altersangaben ist diese Eigenschaft der Reziprokfunktion sehr nützlich, denn man kann in keinem Fall mit Sicherheit den zeitlichen Abstand zwischen dem Schlüpfen der Fische und dem Fangdatum angeben. Vor allem bei den jungen Tieren mit ihren hohen Wachstumsraten wären für sichere Auswertungen exakte Altersangaben erforderlich.

Abgesehen von den zwangsweise pauschalen Altersangaben stellen auch die Mittelwerte der Messungen keine eindeutigen Größen dar, da sie von der zufälligen Zusammensetzung der Stichproben abhängen, die oft eine unzureichende Zahl von Individuen repräsentieren. Bei der unvermeidlichen Unsicherheit der Daten kann man als Ergebnisse der Berechnungen nur Näherungswerte erwarten. Insbesondere scheint dieses die Berechnung des $\xi$-Wertes zu betreffen.

Unter Berücksichtigung dieser Unschärfe der den Auswertungen zugrunde liegenden Daten dürtten die aus den sehr heterogenen Zahlenreihen ermittelten $\xi$-Werte eine befriedigende Übereinstimmung zeigen und als Streuung um einen mittleren SollWert aufzufassen sein, dessen exakte Ermittlung die gegebenen Unterlagen aber nicht gestatten.

Unter diesen Umständen habe ich einheitlich bei den folgenden Ausführungen den Berechnungen als geschätzten mittleren Wert $\xi=0,4$ eingesetzt. Vor allem bei den Daten, die eine geringe Wachstumsspanne umfassen, ergeben sich durch nicht-optimale Werte für $\xi$ nur unwesentlich verschlechterte Näherungen an die Meßdaten. In den meisten Fällen wäre es auch durchaus möglich, das Heringswachstum einfach als Funktion des reziproken Alterswertes (also für $\xi=0$ ) darzustellen, doch sprechen gegen diese Vereinfachung verschiedene Erwägungen.

Die Benutzung eines anderen $\xi$-Wertes hätte zwar für $\mathrm{Y}_{\max }$ und $\log \mathrm{N}$ etwas andere Zahlen ergeben, die grundsätzlichen Ergebnisse dieser Untersuchung würden dadurch aber nicht berührt.

Die Wahl eines einheitlichen Wertes für das additive Alter war deshalb erforderlich, weil der Wert der beiden anderen Parameter der Reziprokfunktion von der Höhe 
des $\xi$-Wertes abhängt und man nur unter dieser Voraussetzung zu Zahlen kommt, die unterschiedliche Meßreihen zu vergleichen gestatten.

\section{DIE GROSSENZUNAHME DER NORDSEEHERINGE}

Die schon beim Vergleich der Tabellen 1 und 2 ausgefallene Zunahme der Maximalwerte spiegelt offensichtlich die in den letzten Jahrzehnten eingetretene Größenzunahme der Nordseeheringe wider. Da dieses Phänomen die Aufmerksamkeit der Fischereibiologen auf sich gezogen hat, verfügen wir in der Literatur über tabellarische Zusammenstellungen, die diese Entwicklung genauer zu verfolgen gestatten. Den weiteren Ausführungen lege ich eine Tabelle von Burd (1962) zugrunde. Die mit der Altersstufe 3 beginnenden und mit der Altersstufe 8 endenden Meßwerte umfassen mit etwa $5 \mathrm{~cm}$ eine zu geringe Zuwachsspanne, um aus ihnen zuverlässige Werte für das additive Alter $\xi$ ermitteln zu können. Hier machen sich aus dem Rahmen fallende Werte zu störend bemerkbar. Aus diesem Grunde habe ich für alle Reihen einheitlich den geschätzten Wert von 0,4 für $\xi$ eingesetzt. Bei der Kürze der Meßreihen und der geringen Zuwachsspanne beeinflußt die Abweichung vom "wahren" Wert die Annäherung an die Daten kaum. Die angegebenen Werte für $s \%$ liegen weit innerhalb des Streubereiches der Meßdaten.

Die Betrachtung von Tabelle 3 zeigt, daß zwischen 1932 und 1951 die Maximalwerte für die Länge im Mittel kurz über $30 \mathrm{~cm}$ liegen, was in bester Übereinstimmung steht mit den Werten, die sich aus den Tabellen von Ford für die Kanalheringe er-

Tabelle 3

Parameter der Reziprokfunktion für das Längenwachstum des Herings unter Zugrundelegung eines einheitlichen $\xi$-Wertes von 0,4 und den Daten yon Burd (1962). (Transversalauswertung)

\begin{tabular}{|cccc|}
\hline Jahr & $\mathrm{L}_{\max }$ & $\log \mathrm{N}$ & so\% \\
\hline 1932 & 30,08 & 0,4186 & 0,62 \\
1933 & 29,68 & 0,3868 & 1,05 \\
1934 & 30,31 & 0,4132 & 0,85 \\
1935 & 29,94 & 0,3803 & 1,01 \\
1936 & 30,18 & 0,3712 & 0,77 \\
1937 & 29,92 & 0,3520 & 0,44 \\
1938 & 30,16 & 0,3616 & 1,03 \\
1939 & 30,79 & 0,4042 & 0,85 \\
1946 & 30,48 & 0,3725 & 0,96 \\
1947 & 30,31 & 0,3464 & 0,46 \\
1948 & 30,45 & 0,3608 & 0,42 \\
1949 & 30,58 & 0,3672 & 0,57 \\
1950 & 30,50 & 0,3470 & 0,80 \\
1951 & 30,60 & 0,3110 & 0,47 \\
1952 & 31,17 & 0,3387 & 1,09 \\
1953 & 31,42 & 0,3540 & 0,73 \\
1954 & 31,97 & 0,3722 & 0,99 \\
1955 & 32,72 & 0,4132 & 1,00 \\
1956 & 33,26 & 0,4773 & 0,86 \\
1957 & 33,67 & 0,4910 & 0,76 \\
1958 & 33,77 & 0,4893 & 0,92 \\
\hline
\end{tabular}


gaben. Der Schwankungsbereich für die ermittelten Maximalwerte erscheint angesichts der unvermeidlichen Unsicherheit der Mittelwerte erstaunlich gering. Ab 1952 tritt dann in den Maximalwerten deutlich die bekannte Steigerung des Wachstums der Nordseeheringe zutage, die wir schon in den Auswertungen der neueren Messungen in Tabelle 2 sahen.

Im Anschluß an die Bezeichnungsweise in der Anthropologie werde ich diese Größenzunahme als Wachstums-Akzeleration bezeichnen. Die Stetigkeit des Vorgangs spricht mehr für eine Anderung der Wachstumseigenschaften der Nordseeheringe als für das Vordringen größerer Heringsrassen.

Im Gegensatz zu der relativen Konstanz der Maximalwerte steht die recht große Variabilität der Geschwindigkeitskonstanten $(\log \mathrm{N}) . \mathrm{Da}$ der $\xi$-Wert in allen Fällen konstant gewählt wurde und die Maximalgrößen bis 1951 praktisch konstant blieben, konzentriert sich bis zu diesem Zeitpunkt die Aussage über den Wachstumsablauf in diesem einen Parameter. Offensichtlich besteht kein fester Zusammenhang zwischen dem Wert von $\log \mathrm{N}$ und der Maximalgröße. Erst nach 1956 ergibt sich eine deutliche Steigerung der Werte für die Geschwindigkeitskonstanten.

Die Tabelle von Burd enthält in den einzelnen Zeilen die Mittelwerte für die in den bezeichneten Jahren gefangenen Heringe, die also verschiedenen Geburtsjahrgängen angehören. Im Anschluß an die Bezeichnung in der Anthropologie möchte ich sie als „Transversalmessungen " bezeichnen. Solche Transversalmessungen liefern nur unter der Bedingung zutreffende Parameterwerte, daß der Wachstumsablauf in den verschiedenen Jahren konstant geblieben ist. Bei Fischen, deren Wachstum durch Umweltfaktoren wie Temperatur und Ernährung stark beeinflußt wird, ist das sicher nicht der Fall. Hinzu tritt noch, daß durch die Akzeleration Jahrgänge mit unterschiedlicher Wachstumstendenz zusammengefaßt werden.

Tabelle 4

Longitudinalauswertung der Werte von BuRD (1962) für das Längenwachstum des Nordsee-Herings $(\xi=0,4)$

\begin{tabular}{|cccc|}
\hline Jahr & $\mathrm{L}_{\max }$ & $\log \mathrm{N}$ & $\mathrm{s} \%$ \\
\hline $1932 / 38$ & 30,72 & 0,4567 & 0,82 \\
$1933 / 39$ & 30,79 & 0,4201 & 0,56 \\
$1946 / 52$ & 31,55 & 0,4354 & 0,55 \\
$1947 / 53$ & 31,56 & 0,4159 & 0,75 \\
$1948 / 54$ & 32,25 & 0,4546 & 0,59 \\
$1949 / 55$ & 32,80 & 0,4891 & 0,71 \\
$1950 / 56$ & 32,73 & 0,4345 & 0,60 \\
$1951 / 57$ & 32,66 & 0,4123 & 0,33 \\
$1952 / 58$ & 33,52 & 0,4665 & 0,46 \\
\hline
\end{tabular}

Es erscheint daher wichtig und notwendig, in "longitudinalen" Auswertungen das Wachstum einzelner Jahrgänge zu verfolgen. Die mehrere Jahrzehnte umfassende Tabelle von Burd bietet auch hierfür eine sehr geeignete Unterlage. Da die Longitudinalauswertungen schräg durch die Tabelle vorgenommen werden, ist verständlicherweise die Zahl der zu gewinnenden Parameterwerte wesentlich geringer. 
Auch die Longitudinalauswertungen in Tabelle 4 lassen die Akzeleration des Wachstums der Nordseeheringe erkennen. Der Maximalwert der Vorkriegsfänge liegt zwar etwas höher, aber immer noch in der Größenordnung der Transversalauswertungen. Eindeutig höher liegen aber die Werte für die Geschwindigkeitskonstanten, was möglicherweise mit der schon in den dreißiger Jahren beginnenden Größenzunahme zusammenhängen kann.

Ganz eindeutig ergibt sich dann aber nach 1946 aus den Maximalwerten die Akzeleration: sie liegen in der gleichen Größenordnung, wie sie sich in Tabelle 2 aus den Nachkriegsfängen ergaben.

Die Erhöhung des Maximalwertes ist nicht mit einer eindeutigen Steigerung des Wertes von $\log \mathrm{N}$ verknüpt. Auffällig ist, daß die Standardabweichungen $(\mathrm{s} \%) \mathrm{im}$ Schnitt niedriger liegen als bei den Transversalauswertungen. Die Longitudinalauswertungen kommen anscheinend dem wahren Verlauf von Wachstumskurven näher als die Transversalauswertungen, die Daten für unterschiedliche Wachstumsabläufe zusammenfassen.

Das überraschende Ergebnis dieser Auswertungen ist, daß trotz der Erfassung einer nur geringen Wachstumsspanne sich bei konstant gehaltenem $\xi$-Wert nicht nur innerhalb dieser Meßreihen gut übereinstimmende Maximalgrößen ergaben, sondern daß diese auch übereinstimmten mit Werten, die Meßreihen ganz anderer Herkunft lieferten.

Die weitgehende Konstanz des rechnerischen Maximalwertes, die sich aus recht unterschiedlich erscheinenden Zahlenreihen ergab, ist nicht an die hier angewandte Reziprokfunktion gebunden, sondern ergibt sich auch für den Maximalwert der BERTALANFFY-Funktion, wie schon die Angaben von Burd (1962) und SCHumacher (1967) erkennen lassen. Ich selbst fand auf dem Wege über die Regressions-Auswertung der Ford-Walford-Formel (Krüger 1973) als Maximalwert für die Daten von BrIDger (1961) für den Nordseehering 27,7-28,4 cm für die Jahre vor 1950; bis 1958 steigen die Werte auf $31,2 \mathrm{~cm}$ an.

Die Maximalwerte der BerTALANFFY-Funktion liegen erwartungsgemäß niedriger als die für die Reziprokfunktion. Der absolute Wert der maximalen Dimension stellt also keine eindeutige Größe dar, sondern hängt von der mathematischen Interpretation der Wachstumskurven ab. Wenn auch beim Hering die rechnerischen Maximalwerte sehr nahe bei realiter beobachteten Fischgrößen liegen, stellen sie nur rein mathematisch $\mathrm{zu}$ deutende Parameter dar.

Tabelle 1 verdeutlicht, daß wir nicht für alle Heringsbestände den gleichen Maximalwert erhalten. Angesichts der Tatsache, daß sich für den Nordseehering aus Messungen verschiedenster Herkunft nur ein sehr enger Schwankungsbereich dieses Parameters ergab, muß man schließen, daß wir es bei den abweichenden Maximalwerten mit anderen Beständen zu tun haben, die sich in ihren Wachstumseigenschaften unterscheiden. Neben den Kanalheringen mit einem Maximalwert von etwa $30,5 \mathrm{~cm}$ erkennt man die skandinavischen Heringe mit einer Maximallänge von $32-33 \mathrm{~cm}$ und die atlantischen Heringe von Island mit einer Maximallänge von $45 \mathrm{~cm}$.

In der auf Messungen aus neuerer Zeit beruhenden Tabelle 2 ist der Unterschied zwischen den Nordsee- und den skandinavischen Heringen als Folge der Wachstumsakzeleration nicht mehr so ausgeprägt. Aus Daten für Heringe von der Georges Bank, 
die mir Herr Dr. Schumacher (Bundesforschungsanstait für Fischerei, Hamburg) freundlicherweise zur Verfügung stellte, errechnet sich die Maximalgröße zu $42 \mathrm{~cm}$. Sie gehören offensichtlich zum atlantischen Bestand.

Anhangsweise seien noch die Maximallängen von Ostseeheringen gegeben. Es errechnen sich aus den Daten von ANw AND (1962) für die Frühjahrsheringe ein Maximalwert von $33,1 \mathrm{~cm}(\xi=0,8)$ und für die Herbstheringe $30,6 \mathrm{~cm}(\xi=0,7)$. Nach den Erfahrungen am Nordseehering dürte dieser Unterschied die Streuung bei der Bestimmung dieses Parameters überschreiten.

Die rechnerische Maximalgröße scheint also eine Möglichkeit zur Unterscheidung von Heringsbeständen zu bieten.

\section{GESCHWINDIGKEITSKONSTANTE UND WENDEPUNKT}

Im Gegensatz zur Maximalgröße, die für konstante $\xi$-Werte zu weitgehend übereinstimmenden Zahlen führte und auch die Akzeleration widerspiegelt, zeigen die Werte für $\log \mathrm{N}$ eine große Variabilität und liefern nicht so deutlich erkennbare Beziehungen.

Die zahlenmäßigen Werte der beiden anderen Parameter sind abhängig von der Höhe des eingesetzten $\xi$-Wertes und ändern sich im gleichen Sinn wie $\xi$. Allerdings wird der Maximalwert von einer Anderung von $\xi$ weniger stark betroffen als die Geschwindigkeitskonstante.

Die Werte von $Y_{\max }$ und $\log \mathrm{N}$ sind aber nicht streng miteinander verknüpt, wie man z. B. Tabelle 3 entnehmen kann. Für das Jahr 1934 ergibt sich für den

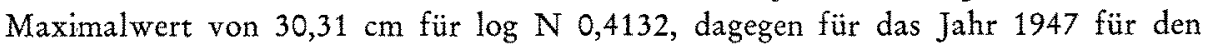
gleichen Maximalwert $\log N=0,3464$. Da der Maximalwert und der vorgegebene $\xi$-Wert identisch sind, finden alle Einflüsse auf das Wachstum, wie z. B. Temperatur und Ernährung, ihren Ausdruck in einem einzigen Parameter.

Für die Aufhellung der hier vorliegenden Verhältnisse erscheint mir die vergleichende Untersuchung von SCHumacher (1967) an Heringen von 3 verschiedenen Fangplätzen der Nordsee aufschlußreich. Der Autor hat mir die seinen Auswertungen zugrunde liegenden Mittelwerte, die er in seiner Arbeit nicht angab, dankenswerterweise zur Verfügung gestellt. Ich wertete sie wieder auf der Basis des $\xi$-Wertes von 0,4 aus. Hierbei blieb die Altersgruppe 2 unberücksichtigt, deren Mittelwerte nicht als ganz repräsentativ angesehen werden können. Die erhaltenen Parameter sind in Tabelle 5 zusammengestellt.

\section{Tabelle 5}

\section{Parameter des Längenwachstums der Heringe aus drei Bereichen der Nordsee} (nach Daten von Dr. Schumacher). $\xi=0,4$

\begin{tabular}{|lccc|}
\hline \multicolumn{1}{|c}{ Fanggebiete } & $\mathrm{L}_{\max }$ & $\log \mathrm{N}$ & s\%/ \\
\hline Nördliche Nordsee (Buchan) & 31,69 & 0,3022 & 0,60 \\
Mittlere Nordsee (Dogger) & 32,32 & 0,4043 & 0,42 \\
Südliche Nordsee (Downs) & 32,21 & 0,4606 & 0,29 \\
\hline
\end{tabular}


Die Maximalwerte für die mittlere und südliche Nordsee stimmen fast vollkommen überein und liegen in der Größenordnung der Nachkriegswerte von BURD (1962) oder PARrish \& CRATg (1957). Der Maximalwert für die Heringe aus der nördlichen Nordsee liegt etwas niedriger. Die Frage, ob dieser Unterschied signifikant ist, muß offenbleiben. Er ist aber so gering, daß wir ihn für unsere Betrachtungen vernachlässigen können und unsere Aufmerksamkeit nur auf die Geschwindigkeitskonstante richten.

Wir sehen bei $\log \mathrm{N}$ eine ganz eindeutige Zunahme des Wertes von Norden nach Süden. Es wäre falsch, hieraus etwa den Schluß zu ziehen, daß sich mit zunehmender Temperatur des Wassers die Wachstumsgeschwindigkeit der Heringe erhöht. Es lassen sich aber am vorliegenden konkreten Beispiel die Beziehungen zwischen dem Zahlenwert der Geschwindigkeitskonstante und dem Wachstumsablauf deutlich machen.

Die Wachstumsgeschwindigkeit - also die Größenzunahme je Zeiteinheit - stellt keine gleichbleibende Größe dar, sondern ist einer stetigen Veränderung unterworfen. Mathematisch beschrieben wird sie durch den Differentialquotienten, der für die Reziprokfunktion lautet:

$$
\frac{\mathrm{dy}}{\mathrm{d} \chi}=\mathrm{y} \frac{\ln \mathrm{N}}{(\chi+\xi)^{2}}
$$

Die auf die jeweilige Größe bezogene relative Wachstumsgeschwindigkeit ist:

$$
\frac{\mathrm{d} y}{\mathrm{y} \cdot \mathrm{d} \chi}=\frac{\ln \mathrm{N}}{(\chi+\xi)^{2}}
$$

Sie liegt der semilogarithmischen Wiedergabe von Wachstumsdaten zugrunde und nimmt - wie man leicht übersieht - mit steigendem $\chi$ kontinuierlich $a b$.

Die linearen Wachstumsraten zeigen dagegen ein anderes Bild. Sie sind sehr gering, solange y klein ist. Mit zunehmenden Werten von y nehmen sie zunächst zu, erreichen aber am Wendepunkt der linearen Wachstumskurve ein Maximum und sinken danach wieder ab, um sich asymptotisch dem Wert Null zu nähern. Die graphische Darstellung der linearen Wachstumsraten liefert eine schiefe Glockenkurve.

Die Lage des Wendepunktes errechnet sich für die Reziprokfunktion nach:

$$
\chi \text { Wendepunkt }=\frac{\ln \mathrm{N}}{2}-\xi \sim 1,15 \log \mathrm{N}-\xi
$$

Während das Alter beim Wendepunkt von der Geschwindigkeitskonstante und dem additiven Zeitwert abhängt, ist die zu diesem Zeitpunkt erreichte Dimension ausschließlich eine Funktion der Maximalgröße:

$$
\mathrm{y}_{\text {Wendepunkt }}=\frac{Y_{\max }}{\mathrm{e}^{2}} \cong \frac{Y_{\max }}{7,4}
$$

$\mathrm{Da}$ wir in den drei gewählten Beispielen auf der Basis des übereinstimmend gewählten $\xi$-Wertes praktisch übereinstimmende Maximalwerte erhielten, bedeutet dieses, daß am Wendepunkt alle 3 Bestände die gleiche Größe haben. Aber die Heringe aus dem Norden mit ihrem kleineren $\xi$-Wert erreichen diese Größe früher als die Heringe aus dem Süden mit ihrem höheren $\xi$-Wert. Bis zum Wendepunkt ist also die Wachstumsgeschwindigkeit der nördlichen Bestände höher als die der südlichen. 
Nach dem Wendepunkt ist das Verhältnis gerade umgekehrt, da alle drei Bestände dem gleichen Endwert zustreben. Die Fische mit dem früher gelegenen Wendepunkt haben einen Wachstumsvorsprung, der nach dem Wendepunkt zu einer Reduzierung der Wachstumsgeschwindigkeit führt und umgekehrt.

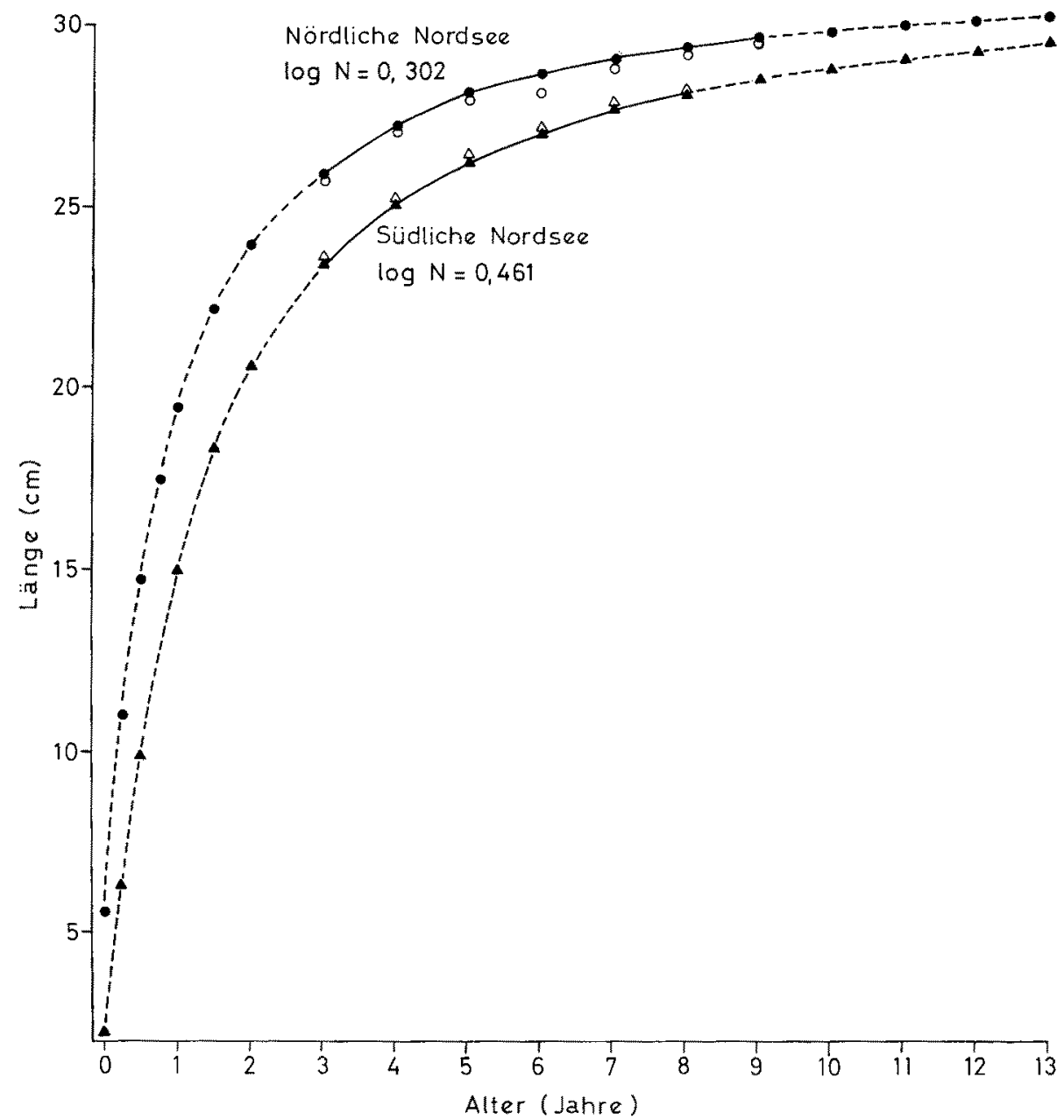

Abb. 1: Vergleich der Wachstumskurven von Heringen aus der südlichen und nördlichen Nordsee unter der Annahme gemeinsamer Werte für $L_{\max }(32 \mathrm{~cm})$ und $\xi(0,4)$. Die nach den Daten von Schumacher (1967) ausgewertete Spanne ist durch ausgezogene Linien gekennzeichnet, der extrapolierte Bereich ist gestrichelt. Berechnete Daten sind durch geschlossene Symbole, gemessene Daten durch offene Symbole wiedergegeben. Durch den für beide Kurven nicht optimalen $L_{\max }$-Wert weichen die eingetragenen Meßpunkte etwas stärker von den hypothetischen Kurven $a b$, die sich nur im Wert von $\log \mathrm{N}$ unterscheiden. Die Heringe aus der nördlichen Nordsee zeichnen sich offensichtlich durch ein stärkeres Wachstum aus, auch wenn in der ausgewerteten Spanne die Zuwachsraten der südlichen Heringe größl ser sind. Die in der Nähe der Altersstufe 0 liegenden Wendepunkte beider Kurven, die in diesem Bereich erheblich von realen Werten abweichen, treten kaum hervor 
Am deutlichsten werden die Verhältnisse in einer graphischen Darstellung (Abb. 1). In ihr sind in Anlehnung an das konkrete Beispiel $32 \mathrm{~cm}$ für $Y_{\max }$ und 0,4 für $\xi$ eingesetzt. Für das Wachstum der nördlichen Bestände ist $\log N=0,3022$ und für die südlichen Bestände $\log N=0,4606$ angenommen.

Die Kurve für die Heringe der nördlichen Population mit ihrem niederen Wert für $\log N$ liegt im ganzen Verlauf oberhalb der Kurve für die Fische aus der südlichen Nordsee. Im ganzen gesehen ist ihre Wachstumsgeschwindigkeit höher, auch wenn sie nach dem Wendepunkt geringer erscheint. Nach dem Wendepunkt nähern sich die Kurven einander.

Dieses Beispiel verdeutlicht die Schwierigkeit einer eindeutigen Beschreibung der Wachstumsgeschwindigkeit. $\mathrm{Da}$ sie sich fortlaufend ändert, kann man sie nur für bestimmte Punkte angeben. Man könnte hierbei an die Halbwertzeit denken, zu der die Fische die halbe Maximalgröße erreicht haben, doch dürfte dieses keine günstige Lösung darstellen.

Eine formal sehr ansprechende Lösung ergibt sich für die am Wendepunkt erw reichte maximale Wachstumsgeschwindigkeit, wenn man in Gleichung (4a) die Gleichung (5) und (6) einsetzt:

$$
\frac{\mathrm{d} \text { YWendepunist }}{\mathrm{d} \chi \text { Wendepunkt }}=\frac{\mathrm{Y}_{\max } \cdot 4}{\ln \mathrm{N} \cdot \mathrm{e}^{2}}=\frac{\mathrm{Y}_{\max }}{4,25 \cdot \log \mathrm{N}}
$$

Dieser Ausdruck besitzt den Vorzug, daß das additive Alter ausgeschaltet, also von der speziellen Form des Kurvenverlaufs unabhängig ist. Für unseren speziellen Fall liefert der Quotient für die Heringe aus der nördlichen Nordsee zutreffend einen höheren Wert als für die südlicher lebenden Fische.

\section{DAS GEWICHTSWACHSTUM}

Die Reziprokfunktion kann in unveränderter Form auch zur Darstellung von Daten für das Gewichtswachstum benutzt werden. Diese Eigenschaft beruht darauf, daß sie einen Wendepunkt besitzt, der im allgemeinen in Gewichtskurven auftritt.

BRIDGER (1961) gibt in seiner Tabelle den Längenwerten von BURD zugeordnete Gewichtsangaben. Der Versuch für sie $\xi$-Werte zu berechnen, führte zu vollkommen unwahrscheinlichen Ergebnissen. Offensichtlich stören die durch die Entwicklung der Geschlechtsprodukte hervorgerufenen Anderungen der Körperproportionen eine regelmäßige Gewichtszunahme. Aus diesem Grunde wurde - wie bei den Auswertungen des Längenwachstums - ein $\xi$-Wert von 0,4 eingesetzt.

Im Prinzip finden sich die gleichen Verhältnisse, wie beim Längenwachstum (Tab. 6). Bis 1949 ergeben sich Maximalwerte gleicher Größenordnung, allerdings ist ihr Schwankungsbereich breiter. Hierin kommt wohl zum Ausdruck, daß das Gewichtswachstum stärker von den Ernährungsverhältnissen abhängig ist. Ab 1950 beobachten wir dann eine deutliche Zunahme der Maximalgewichte und in diesem Fall auch für die Werte von $\log$ N. Die Standardabweichungen sind durchweg höher als für die Längenauswertungen, liegen aber sicher noch innerhalb des Streubereichs der Einzelmessungen. 
Tabelle 6

Parameter der Reziprokfunktion für das Gewichtswachstum der Heringe und Exponent $\beta$ der allometrischen Längen-Gewichts-Beziehung. Daten von BRIDGER (1961). $\xi=0,4$

\begin{tabular}{|cccccrc|}
\hline Jahr & $\mathrm{W}_{\max }$ & $\log \mathrm{N}_{\mathrm{w}}$ & $\mathrm{s} \%$ & $\log \mathrm{N}_{\mathrm{l}}$ & $\log \mathrm{N}_{\mathrm{w}}$ & \\
\cline { 6 - 7 } & & & & & $\log \mathrm{N}_{\mathrm{l}}$ & $\beta$ \\
\hline 1935 & 225,90 & 1,1854 & 2,37 & 0,3803 & 3,117 & 3,072 \\
1936 & 215,44 & 1,0632 & 1,95 & 0,3712 & 2,864 & 2,832 \\
1937 & 235,81 & 1,0705 & 1,74 & 0,3520 & 3,041 & 3,030 \\
1938 & 216,25 & 1,0683 & 3,09 & 0,3616 & 2,954 & 2,948 \\
1946 & 197,99 & 0,8149 & 1,79 & 0,3725 & 2,188 & 2,177 \\
1947 & 233,17 & 0,9212 & 1,99 & 0,3464 & 2,659 & 2,655 \\
1948 & 186,11 & 0,7360 & 1,95 & 0,3608 & 2,040 & 2,042 \\
1949 & 224,32 & 1,0632 & 2,81 & 0,3672 & 2,895 & 2,905 \\
1950 & 249,72 & 1,1103 & 2,80 & 0,3470 & 3,200 & 3,035 \\
1951 & 247,66 & 0,9706 & 2,89 & 0,3110 & 3,121 & 3,138 \\
1952 & 267,84 & 1,1253 & 3,84 & 0,3387 & 3,322 & 3,322 \\
1953 & 278,38 & 1,1345 & 3,04 & 0,3540 & 3,205 & 3,213 \\
1954 & 299,89 & 1,2211 & 4,10 & 0,3722 & 3,281 & 3,293 \\
1955 & 302,98 & 1,2507 & 4,70 & 0,4132 & 3,027 & 3,049 \\
1956 & 329,92 & 1,4924 & 2,80 & 0,4773 & 3,127 & 3,127 \\
1957 & 317,14 & 1,4453 & 2,11 & 0,4910 & 2,944 & 2,941 \\
\hline
\end{tabular}

Die Zahlen für die Geschwindigkeitskonstante haben als solche wenig Aussagewert; auffällig ist nur ihr Absinken zwischen 1946 und 1948, in welchen Jahren sie abweichend von den anderen Jahren deutlich unter 1 liegen.

Aussagewert erhalten die Geschwindigkeitskonstanten für das Gewicht, wenn man sie in Beziehung setzt zu denen des Längenwachstums, indem man den Quotienten $\frac{\log N_{w}}{\log N_{1}}$ bildet. Dieser Quotient stellt nämlich den Exponenten $\beta$ der allometrischen Funktion für die Beziehung zwischen Längen und Gewicht dar: $w=a l^{\beta}$. Die allometrische Formel gestattet unter Ausschaltung des Alters eine Beziehung zwischen diesen beiden Dimensionen herzustellen. Dieser Exponent wurde direkt aus den angegebenen Längen- und Gewichtsdaten für die einzelnen Jahre berechnet und neben den Quotienten der Geschwindigkeitskonstanten gesetzt. Die Werte für $\log \mathrm{N}_{\mathrm{l}}$ für das Längenwachstum sind der Tabelle 3 entnommen. Man erkennt, daß beide Ausdrüdke für die Längen-Gewichts-Beziehung durchweg befriedigend übereinstimmen. Es ergaben sich Werte, die in der Nähe von 3 liegen. Eine Ausnahme bilden die Jahre zwischen 1946 und 1948, in denen die Heringe im Verhältnis zu ihrer Länge zu leicht waren.

ILES (1968) fand für die Jahre zwischen 1943 und 1947 eine deutliche Abnahme der Wachstums-Indices für die O-Gruppe und führt sie auf den crowding-Effekt zurück, der durch die mangelnde Befischung während der Kriegsjahre bedingt war. Man darf hierzu wohl die am allometrischen Exponenten $\beta$ festgestellte Gewichtsabnahme in Parallele stellen.

In der Reziprokfunktion kommt die herabgesetzte Gewichtszunahme in der Verminderung des Wertes von $\log \mathrm{N}$ zum Ausdruck. 1946 und 1948 kommt es auch zu einer Verminderung des Maximalgewichtes, obgleich - wie wir sahen - eine strenge Koppelung $\mathrm{z}$ wischen diesen beiden Parametern nicht besteht. 
Die Parameter für das Längenwachstum (Tab. 3) heben sich für diese Zeit nicht von den übrigen Werten ab. Das abweichende Ergebnis von ILES dürfte darauf zurüdkzuführen sein, daß er seinen Auswertungen nicht direkte Längenmessungen zugrunde legte, sondern von dem Abstand der Jahresringe in den Schuppen auf die jeweiligen Fischlängen schloß. Hier scheinen Besonderheiten der Ringbildung eine Rolle gespielt zu haben.

Im Gegensatz zum Längenwachstum dürften - wie sich hier zeigte und verständlich ist - die Parameter für das Gewichtswachstum Aussagen über die Ernährungsbedingungen zulassen.

\section{DISKUSSION}

Die Auswertung der Wachstumsdaten von Heringsbeständen verschiedenster Herkunft ergab - soweit sie die für exaktere Parameterbestimmungen erforderlichen Altersstufen 0 und 1 enthielten - für das additive Alter der Reziprokfunktion durchweg Werte unter 1. Man hat den Eindruck, daß die rechnerische Bestimmung des $\xi$-Wertes

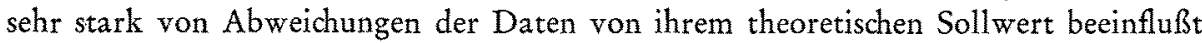
wird. Vermutlich hängt das damit zusammen, daß die Funktion gegen Abweichungen von seinem optimalen Wert recht unempfindlich ist, worunter die Schärfe der Bestimmungen leidet. Man kann aber mit Sicherheit sagen, daß der Wert für das additive Alter $\xi$ zumindest für die Nordseeheringe zwischen 0 und 1 liegt. Dieser Wert ist im Vergleich zu anderen Fischen sehr niedrig. Er übernimmt in der Reziprokfunktion die Aufgabe der Beschreibung der Krümmung der Wachstumskurve, und ein niederer Wert besagt, daß wir es mit einer im Anfangsteil stark ansteigenden und stark gekrümmten Kurve zu tun haben.

Angesichts der Unsicherheit bei der Bestimmung des $\xi$-Wertes erscheint die Übereinstimmung der erhaltenen Zahlen befriedigend, und man darf die Existenz eines einheitlichen Wertes für die Beschreibung des Wachstums der meisten Heringspopulationen annehmen, auch wenn es noch nicht möglich ist, diesen Sollwert festzulegen. Dieser Befund bestätigt meine früher geäußerte Vermutung, daß es sich bei dem additiven Alterswert $\xi$ um einen Parameter handelt, der nicht nur der mathematischen Beschreibung einzelner Zahlenreihen dient, sondern für systematische Kategorien charaktristisch ist. Diese Annahme ist durchaus verständlich, da ohne $Z$ weifel der in den Kurven wiedergegebene Wachstumsablauf ein generisch festgelegtes Merkmal darstellt, und daher wird man auch für den Krümmungsparameter $\xi$ übereinstimmende Werte erwarten dürfen.

Der wirkliche Wert für den Krümmungsparameter konnte - wie gesagt - noch nicht festgelegt werden, da aber die Höhe der beiden anderen Parameter der Funktion von dem eingesetzten $\xi$-Wert abhängt, war es erforderlich, bei den Auswertungen der verschiedenen Zahlenreihen einen einheitlichen Wert einzusetzen. Als solcher diente ein geschätzter mittlerer Wert von 0,4 , der in allen Fällen eine gute Annäherung an die Meßdaten lieferte. Bei der weitgehenden Anpassungsfähigkeit der Reziprokfunktion an nicht optimale Werte von $\xi$ erscheint dieses Vorgehen zulässig.

Im Gegensatz zu der Unsicherheit, die sich aus der Unschärfe der Meßdaten bei $\operatorname{der} \xi$-Bestimmung ergab, steht die Konstanz der Maximalwerte, die sich - wenigstens 
für die Messungen vor dem Kriege - aus den gleichen Reihen für ein festgelegtes $\xi$ ergab. Zum Teil mag hierbei eine Rolle spielen, daß die Höhe des Maximalwertes sich bei Anderung des $\xi$-Wertes relativ nur wenig verschiebt, außerdem bewirkt wohl die angewandte Regressionsberechnung einen Ausgleich zwischen den vom Soll-Wert abweichenden Daten.

Der Maximalwert stellt also offensichtlich ebenfalls ein genetisch festgelegtes Merkmal dar, das aus Wachstumsdaten relativ leicht und sicher zu bestimmen ist und vermutlich als Unterscheidungsmerkmal für Heringspopulationen dienen kann. Es ist eine Frage der Definition, ob man Bestände mit abweichenden Maximalwerten als Rassen zu bezeichnen hat. Die Erhöhung des Maximalwertes bei den Nachkriegsfängen erklärt sich leicht durch die in dieser Zeit erfolgte Größenzunahme der Nordsee-heringe.

Offen bleibt unter diesem Gesichtspunkt die Frage, ob man den von Schumacher (1967) erfaßten Heringsbestand aus der nördlichen Nordsee, der gegenüber den südlicher lebenden Artgenossen eine $6 \mathrm{~mm}$ geringere Maximalgröße ergab, als eine gesonderte Population zu betrachten hat. Dieser Bestand unterscheidet sich auch durch eine statistisch gesicherte höhere Zahl von Kiemenreusenfortsätzen von den weiter südlich lebenden Heringen. Da aber die Zahl der Kiemenreusenfortsätze nach den Ergebnissen von SCHUMAcrier von der Körpergröße abhängt, kann man daran denken, daß bei den bedeutend größeren Jugendstadien der nördlichen Heringe mehr Kiemenreusenfortsätze angelegt werden als bei den Artgenossen aus dem Süden. Man hätte es dann nicht mit einem grundsätzlichen Unterscheidungsmerkmal zu tun und wir hätten in der Nordsee einen einheitlichen Heringsbestand anzunehmen.

Gemäß der mathematischen Definition stellt der Maximalwert eine rein fiktive Zahl dar, und es ist erstaunlich, mit welcher Konstanz die verschiedenen Meßreihen auf diesen irrealen Punkt zustreben, auch wenn die Ausgangsgrößen unterschiedlich gewesen sind.

Es ist also nicht eine realisierte Endgröße, dem das Wachstum zustrebt, sondern eine darüber hinausgehende extrapolierte Dimension. Diese Schlußfolgerung ist nicht an die Reziprokfunktion gebunden, sondern ergibt sich in gleicher Weise aus der BertalanfFy-Funktion. Allerdings stimmen die Maximalwerte der beiden Funktionen nicht überein, so daß man nicht mit Sicherheit sagen kann, auf welchen Maximalpunkt das Wachstum der Heringe tendiert.

Diese Ausrichtung des Wachstums auf einen genetisch festgelegten Maximalwert setzt einen Kontrollmechanismus voraus, der zu jedem Zeitpunkt die erreichte Dimension mit dem Soll-Wert vergleicht und entsprechende Regelmechanismen auslöst. Letztere Folgerung ist durch zahlreiche Beobachtungen an Mensch und Tier bestätigt.

Nun darf man hieraus aber nicht schließen, daß der gesamte Wachstumsablauf von vornherein fest determiniert ist. Es ergaben sich ja auch erhebliche Größenunterschiede vor allem in den ersten Altersstufen. Sie entstehen offensichtlich in der Zeit der exponentiell ansteigenden Wachstumsraten vor dem Wendepunkt der Wachstumskurve, der vermutlich in Abhängigkeit von der Temperatur früher oder später erreicht wird, zeitlich also nicht festgelegt ist.

Bemerkenswert ist hierbei, daß die Wachstumsgeschwindigkeit in der Zeit vor dem Wendepunkt im kälteren Milieu höher ist als in wärmerer Umgebung. Da das 
Wachstum ein Gleichgewicht zwischen auf- und abbauenden Prozessen - Anabolismus und Katabolismus - darstellt, könnte man dieses Phänomen dadurch deuten, daß beide Prozesse temperaturabhängig sind, aber der Katabolismus durch tiefere Temperaturen stärker gehemmt wird als der Anabolismus. Unter dieser Bedingung würde die Wachstumsgeschwindigkeit im kälteren Milieu höher sein.

Die zeitliche Lage des Wendepunktes ist also durch äußere Faktoren zu beeinflussen und wirkt auf diesem Wege auf den Kurvenabschnitt mit abnehmenden exponentiellen Wachstumsraten nach dem Wendepunkt. Ein früher Wendepunkt wirkt in diesem Kurventeil verlangsamend, ein später Wendepunkt beschleunigend auf die Wachstumsgeschwindigkeit.

Die bei einem bestimmten Alter nach dem Wendepunkt erreichte Größe hat zwar Einfluß auf das folgende Wachstum, entscheidend bleibt in dieser Periode aber der Maximalwert als Zielgröße. Trotzdem bleibt die aus der mathematischen Analyse gewonnene Einsicht wichtig, daß für das Wachstums des Herings die frühe Entwicklung von entscheidender Bedeutung ist. Sie liegt auch den Bemühungen von Iles (1967) zugrunde, zu einer quantitativen Erfassung des Heringswachstums zu kommen. Er benutzt hierfür das Wachstum der Altersgruppen 0 und 1, deren Körperlängen er aus den Abständen der Jahresringe in den Schuppen schätzt.

Der Einfluß der Ausgangsgröße $\left(\mathrm{L}_{0}\right)$ auf das Wachstum ist auch in der ursprünglichen Fassung der BERTALANFFY-Funktion (1934) enthalten:

$$
1_{\chi}=\mathrm{L}_{\infty}-\left(\mathrm{L}_{\infty}-\mathrm{L}_{0}\right) \cdot \mathrm{e}^{-\mathrm{K}} \cdot \chi
$$

In der von Beverton \& HoLT (1957) vorgeschlagenen und heute zumeist angegebenen Schreibung tritt an die Stelle von $\mathrm{L}_{0}$ als dritter Parameter der additive Zeitwert $\tau_{0}$ :

$$
\mathrm{l}_{\chi}=\mathrm{L}_{\infty}\left(1-\mathrm{e}^{-\mathrm{K}\left(\chi-\tau_{0}\right)}\right)
$$

Die Auswertung der Daten von Schumacher (1967) auf der Basis der Ford-WaLFORD-Formel lieferte für die BERTALANFFY-Parameter sehr nahe beieinanderliegende Werte für die Maximalgröße und die Geschwindigkeitskonstante $\mathrm{K}$, dagegen deutliche Differenzen für $\mathrm{L}_{0}$ bzw. $\tau_{0}$. Allerdings ließen sich die Beziehungen nicht ganz so deutlich erkennen, wie bei der Reziprokfunktion. Die BerTALANFFY-Funktion enthält also die Anfangsgröße als eigenen Parameter, da sie aber keinen Wendepunkt enthält, kann sie nur den nach dem Wendepunkt liegenden Kurventeil beschreiben und daher auch keinen Aufschluß darüber geben, wie es zu den unterschiedlichen Ausgangsgrößen kommen kann.

Wenn ich meine Ausführungen über das - im Grunde lange bekannte - gegensätzliche Verhalten der Wachstumsgeschwindigkeit vor und nach dem Wendepunkt auf den Wendepunkt der Reziprokfunktion bezog, mag das ein gewisses Bedenken erregen, denn die Lage des Wendepunktes errechnet sich für die Zeit kurz nach dem Schlüpfen oder sogar noch davor. Zu diesem Zeitpunkt haben die aus dem adulten Wachstum errechneten Parameter sicherlich keine Gültigkeit mehr.

Hierzu ist zu bemerken, daß das Längenwachstum eine abgeleitete Dimension darstellt, da das Grundphänomen des Wachstums die Vermehrung der lebenden Masse darstellt und für das Gewichtswachstum liegt der Wendepunkt wesentlich später. Andererseits ändern sich für das Längenwachstum die Verhältnisse nicht, wenn wir an die 
Stelle der hohen Wachstumsgeschwindigkeit vor dem Wendepunkt ein starkes larvales Wachstum setzen, das nur anderen Parametern folgt. Es tritt auch dann in der Wachstumskurve ein Wendepunkt auf. Dem von mir ausgewerteten Wendepunkt für das Längenwachstum der Heringe kommt vermutlich nur formale Bedeutung zu, trotzdem erscheint er mir für das Verständnis des ganzen Wachstumsablaufs äußerst bedeutungsvoll. Aus diesem Grunde ist die Reziprokfunktion, die einen Wendepunkt einschließt, nicht nur für die Beschreibung, sondern auch für die Interpretation von Wachstumsvorgängen besser geeignet als die BERTALANFFY-Funktion, die keinen Wendepunkt enthält und nur für die Wiedergabe von Kurven mit fallenden Wachstumsraten geeignet ist.

Die durch die mathematische Auswertung des Heringswachstums gewonnenen Aufschlüsse fügen sich zwanglos in die bisher gewonnenen Erkenntnisse ein und es ist zu erwarten, daß ihre Anwendung in Zukunft diese Einsichten ergänzen und erweitern wird. Bei der großen Zahl der in der Literatur vorliegenden Wachstumsdaten für den Hering war die Auswahl einer begrenzten Zahl von Beispielen erforderlich, um die prinzipiellen Möglichkeiten dieses Verfahrens ausführlicher erörtern zu können. Die große Zahl der Unterlagen bot die Möglichkeit, grundsätzliche Fragen der Wachstumsmathematik an diesem konkreten Beispiel zu erörtern und zu klären. Es sei hier vor allem an die Frage der Darstellung der Wachstumsgeschwindigkeit gedacht.

Geeignete mathematische Modelle stellen eine unerläßliche Voraussetzung für ein tieferes Eindringen in das Verständnis des Wachstums dar, das ja eines der elementaren Phänomene des Lebens bildet.

Aber auch für zahlreiche spezielle Fragen sind mathematische Wachstumsmodelle von größter Bedeutung. Dadurch, daß sie den Inhalt umfangreicher und unübersichtlicher Tabellen in wenigen Parametern zusammenfassen, die den Wachstumsablauf charakterisieren, liefern sie Unterlagen für exakte Vergleiche oder für Fragen der Populationsdynamik.

Wesentlich ist hierbei, daß der Aussagewert der Parameter geklärt und an konkreten Beispielen getestet ist. Wichtig ist aber auch, daß die Modelle relativ einfach zu handhaben sind, eine Forderung, die mit den modernen Elektronen-Rechnern leicht $\mathrm{zu}$ erfüllen ist.

\section{ZUSAMMENFASSUNG}

1. Die Parameter der vom Autor vorgeschlagenen "Reziprokfunktion" werden für das Wachstum von Heringen aus älteren und jüngeren Messungen bestimmt. Diese Funktion lautet in logarithmischer Form:

$$
\log \mathrm{y}_{\mathrm{x}}=\log \mathrm{Y}_{\mathrm{max}}-\frac{1}{x+\xi} \log \mathrm{N}
$$

2. Für das additive Alter $(\xi)$ werden Werte unter 1 gefunden. Für die weiteren Auswertungen wird ein geschätzter mittlerer Wert von 0,4 eingesetzt.

3. Meßreihen aus der Zeit vor 1940 ergeben für den Maximalwert $\left(L_{\max }\right) \mathrm{ca} .30 \mathrm{~cm}$. In späteren Jahren erhöhte sich der Wert auf ca. $34 \mathrm{~cm}$. 
4. Die skandinavischen und atlantischen Heringe unterscheiden sich von den Nordseeheringen durch höhere Maximalwerte.

5. Die Werte für die Geschwindigkeitskonstante $(\log N)$ können bei gleichen Werten von $\xi$ und $Y_{\max }$ unterschiedlich sein.

6. Die Geschwindigkeitskonstante kennzeichnet die Lage des Wendepunktes der Wachstumskurve. Die Dimension beträgt zu diesem Zeitpunkt $\frac{Y_{\max }}{7,4}$, hängt also nur von der Maximalgröße ab.

7. Für die drei von Schumacher (1967) untersuchten Populationen des Nordseeherings ergeben sich steigende Werte für die Geschwindigkeitskonstante von Norden nach Süden.

8. Ein niedriger Wert für $\log \mathrm{N}$ kennzeichnet eine frühe Lage des Wendepunktes und eine hohe Wachstumsgeschwindigkeit vor diesem Punkt und umgekehrt.

9. Da das Wachstum der 3 Heringspopulationen einem annähernd gleichen Maximalwert zustrebt, folgt, daß eine hohe Wachstumsgeschwindigkeit vor dem Wendepunkt durch eine geringere Geschwindigkeit nach diesem Punkt ausgeglichen wird.

10. Die höchste Wachstumsgeschwindigkeit am Wendepunkt ist durch den Ausdruck $\frac{Y_{\max }}{4,25 \cdot \log N}$ gegeben. Möglicherweise bietet er ein nützliches Hilfsmittel für den quantitativen Vergleich von Wachstumsgeschwindigkeiten.

Danksagung. Die Auswertungen erfolgten auf dem Klein-Computer $700 \mathrm{~B}$ der Fa. WANG, der mir dankenswerterweise von der Deutschen Forschungsgemeinschaft zur Verfügung gestellt wurde.

\section{ZITIERTE LITERATUR}

ANwAND, K., 1962-1963. Das Wachstum der Frühjahrs- und Herbstheringe aus den Gewässern um Rügen. Z. Fisch. 11, 201-209.

BAgenal, T. B., 1955. The growth rate of the long rough dab Hyppoglossoides platessoides. J. mar. biol. Ass. U. K. 34, 297-311.

BertalanfFy, L. von, 1934. Untersuchungen über die Gesetzlichkeit des Wachstums. I. Allgemeine Grundlagen der Theorie. Wilhelm Roux Arch. EntwMech. Org. 131, 613-653.

Beverton, R. J. \& Holt, S. J., 1957. On the dynamics of exploited fish populations. Fishery Invest., Lond. (Ser. 2) 19, 1-533.

Bridger, J. P., 1961. On fecundity and larval abundance of Downs herring. Fishery Invest., Lond. (Ser. 2) 23, 1-30.

Burd, A. C., 1962. Growth and recruitment in the Herring of the southern North Sea. Fishery Invest., Lond. (Ser. 2) 23, 1-42.

Ford, E., 1933. An account of the herring investigations conducted at Plymouth during the years from 1924-1933. J. mar. biol. Ass. U. K. 19, 305-384.

Heincke, F., 1898. Naturgeschichte des Herings. Abh. dt. Seefisch.Ver. 2.

Hohendorf, K., 1966. Eine Diskussion der BertalanfFy-Funktion und ihre Anwendung zur Charakterisierung des Wachstums von Fischen. Kieler Meeresforsch. 22, 70-97.

ILEs, T. D., 1967. Growth studies on North Sea herring. I. The second year's growth (I-group) of East Anglian herring, 1939-63. J. Cons. perm. int. Explor. Mer, 31, 56-76.

- 1968. Growth studies on North Sea herring. II. O-group growth of East Anglian herring. J. Cons. perm. int. Explor. Mer, 32, 98-116.

KrügER, F., 1962. Über die mathematische Darstellung des tierischen Wachstums. Naturwissenschaften 49,454 . 
- 1965. Zur Mathematik des tierischen Wachstums. I. Grundlagen einer neuen Wachstumsfunktion. Helgoländer wiss. Meeresunters. 12, 78-136.

- 1967. BertalanfFy-Funktion und Ford-WaLFord-Formel. Helgoländer wiss. Meeresunters. 19, 193-206.

- 1973. Zur Mathematik des tierischen Wachstums. II. Vergleich einiger Wachstumsfunktionen. Helgoländer wiss. Meeresunters. 25, 509-550.

Parrish, B. B. \& CRAIG, R. E., 1957. Recent changes in North Sea herring fisheries. Rapp. P.-v. Réun. Cons. perm. int. Explor. Mer 143, 12-24.

- \& SAville, A., 1965. The biology of the North-East Atlantic herring populations. Oceanogr. mar. Biol. 3, 323-373.

Thambs-Lyche, H., 1965. Herring stock record data. Statist. Newsl. Cons. perm. int. Explor. Mer 31, 1-112.

SChUmaCher, A., 1967. Bestandskundliche Untersuchungen am Nordseehering (Clupea harengus). Ber. dt. wiss. Komm. Meeresforsch. 19, 13-63.

Anschrift des Autors: Prof. Dr. F. KRÜGER

Biologische Anstalt Helgoland (Zentrale)

2 Hamburg 50

Palmaille 9

Bundesrepublik Deutschland 